\title{
LUIS MOYA Y LAS BÓVEDAS TABICADAS EN LA POSGUERRA ESPAÑOLA
}

\author{
(LUIS MOYA AND TILE VAULTS IN POST-WAR SPAIN)
}

Josep Ma Adell

Profesor, Doctor Arquitecto. Universidad Politécnica Madrid (UPM). Dpto. Construcción y Tecnología Arquitectónicas (DCTA). Escuela Técnica Superior de Arquitectura (ETSAM). Director Línea Investigación sobre fábricas en el Grupo de Investigación de Tecnologías Innovadoras y Sostenibles en Edificación (TISE-fábricas)

Antonio Rolando

Doctor Arquitecto. Profesor Titular. UPM-DCTA-ETSAM-TISE

ESPAÑA

Fecha de recepción: 2- III-05

\author{
ESPANA
}

\section{RESUMEN}

Después de la Guerra Civil, y ante la precaria situación económica del país, se volvió a retomar en España, la construcción tabicada como medio de economizar acero en la edificación, y aprovechando la mano de obra barata del momento.

Luis Moya destacó en este aspecto, reinvestigando sobre la construcción tabicada siendo perfectamente conocedor de la obra de los Guastavino en Nueva York, de principios de siglo, por sus visitas realizadas acompañadas del Profesor Collins a EEUU.

La Arquitectura de Moya destacó por aplicar soluciones de bóvedas tabicadas de mediana luz que normalmente reforzaba empleando arcos tabicados internos vistos, o bien acostillando las bóvedas por su trasdós.

Esta labor arquitectónica basada en su propia experimentación constructiva, la publicó en el libro "Bóvedas Tabicadas", siendo uno de los pocos tratados amplios sobre este tema existente, superando con él a lo publicado por Fornés-Gurrea y Bergós con anterioridad.

\begin{abstract}
SUMMARY
In the precarious economic conditions prevailing after the Spanish Civil War, tiled vault construction was resumed to economize on steel and capitalize on the cheap labour available at the time.
\end{abstract}

Luis Moya excelled in this area, re-exploring this construction technique. Moya benefited in this regard from his thorough knowledge of the early twentieth century works of the Guastavinos in New York acquired during his visits to the U.S.A. with Professor Collins.

His architecture featured tiled vaults with medium-sized spans, normally reinforced with exposed internal Basque tiled arches or ribbing on the extrados.

He described his architectural experimentation in this field in a book titled "Bóvedas tabicadas" (tile vaults), one of the few extensive treatises on the subject, which surpassed the papers published earlier by Fornés-Gurrea and Bergós.

\section{ANÁLISIS DEL LIBRO "BÓVEDAS TABICADAS"}

Siguiendo los apartados del libro "Bóvedas Tabicadas", y considerando algunas de sus figuras, destacamos lo más significativo:

\section{- Clases de bóvedas}

En este capítulo expone gráficamente los diversos trazados de las distintas bóvedas.
En todos los casos, están hechas con una primera vuelta de rasilla con yeso, y de una o varias de rasilla con mortero de cemento, disponiendo siempre los ladrillos de plano (Fig. 2). Desarrolla distintos tipos de bóvedas: cilíndrica (Fig. 3), esférica (Fig. 4), bóveda por arista peraltada (Fig. 6 y Figs. 7 y 8), lunetos, destacando la disposición de las cerchas para generar las diversas formas y de las cuerdas o alambres para marcar la disposición de los ladrillos de las vueltas. 


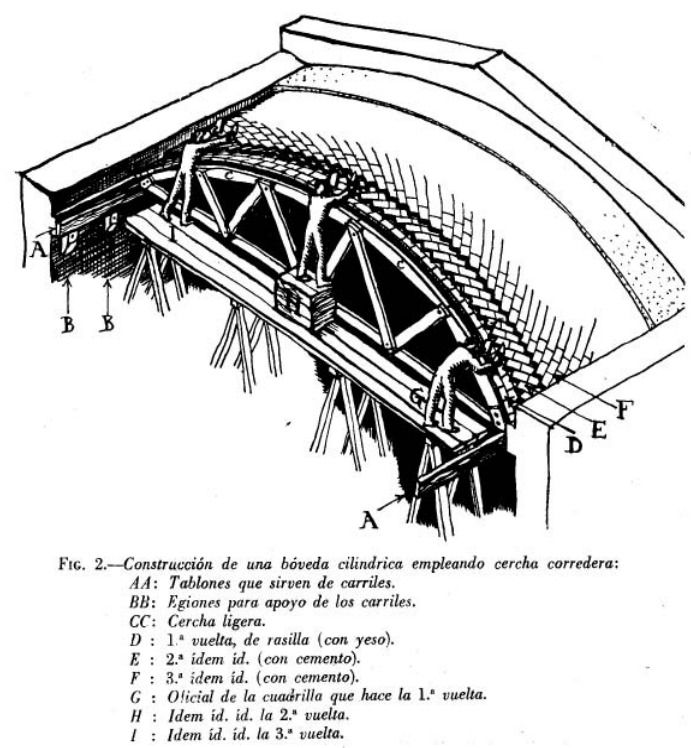

Fig. 2

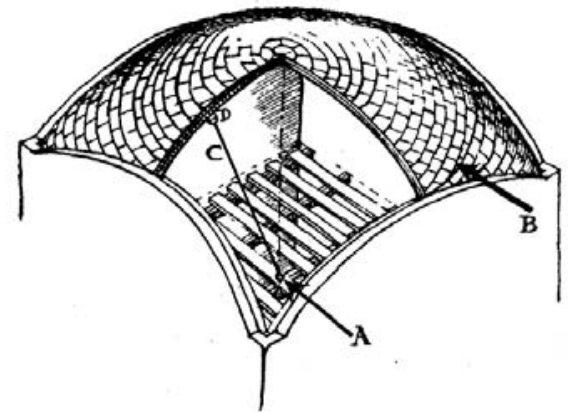

Figs. 4 y 5.-Construcción de una bóveda esférica sin cercha: A, Centro de le esfera con una anilla pa. ra atar la cuerda; B, Principio de la espiral; C, Cuerda; D, Tope coloca to a la distancia $A D$

Fig. 4

\section{- Construcción}

En el primer apartado de este capítulo sobre "despiezo", expone, de forma gráfica, con claridad, las ventajas constructivas y de economía de material, a la hora de disponer los ladrillos en las bóvedas tabicadas, con respecto a las dovelas de las bóvedas de piedra (Fig. 12).

En los apartados la "marcha de la construcción" y el "perfil de la bóveda", refiere las relaciones geométricas entre la luz y la flecha de las distintas bóvedas empleadas.

Dedica en este capítulo un apartado sobre el "arranque en voladizo" de las bóvedas (atirantadas o no), con sus variantes en hiladas de ladrillo macizo en voladizo, o arranque sobre imposta sobresaliente (Fig. 16).

Especial atención tiene el apartado dedicado a "bóvedas nervadas", donde desarrolla su especialidad con nervadu-

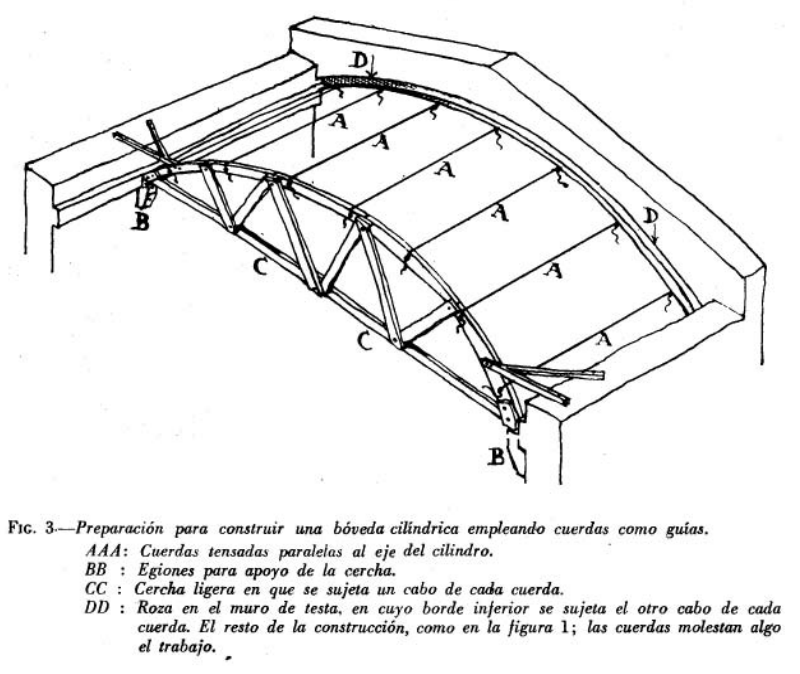

Fig. 3

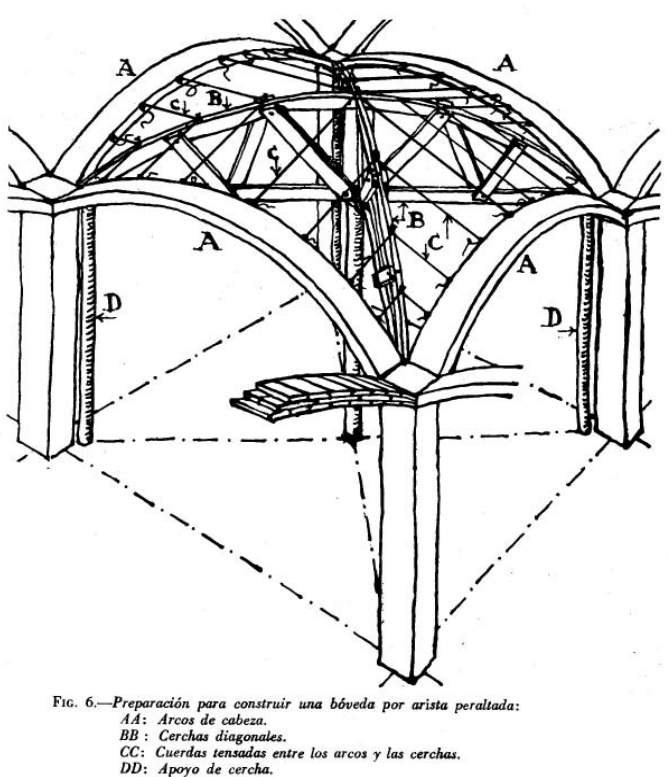

Fig. 6

ras de arcos cruzados, que dejan un hueco central en la cubierta para disponer sobre él una linterna que ilumine el espacio (Fig. 19).

\section{- Cálculo de bóvedas}

El apartado dedicado al cálculo se basa solamente en algunas cuestiones de proporciones geométricas.

\section{. Empujes}

Es de vital importancia en las construcciones donde se disponen bóvedas, soportar los empujes que éstas generan, para lo que puede recurrirse a secuencias de bóvedas que transmiten sus esfuerzos respectivos entre sí hasta una zona de arriostramiento (Fig. 25 y Fig. 42) o, por el contrario, absorber el empuje de las bóvedas empleando tirantes capaces de soportar los correspondientes esfuerzos a tracción (Fig. 36 y Fig. 37). 

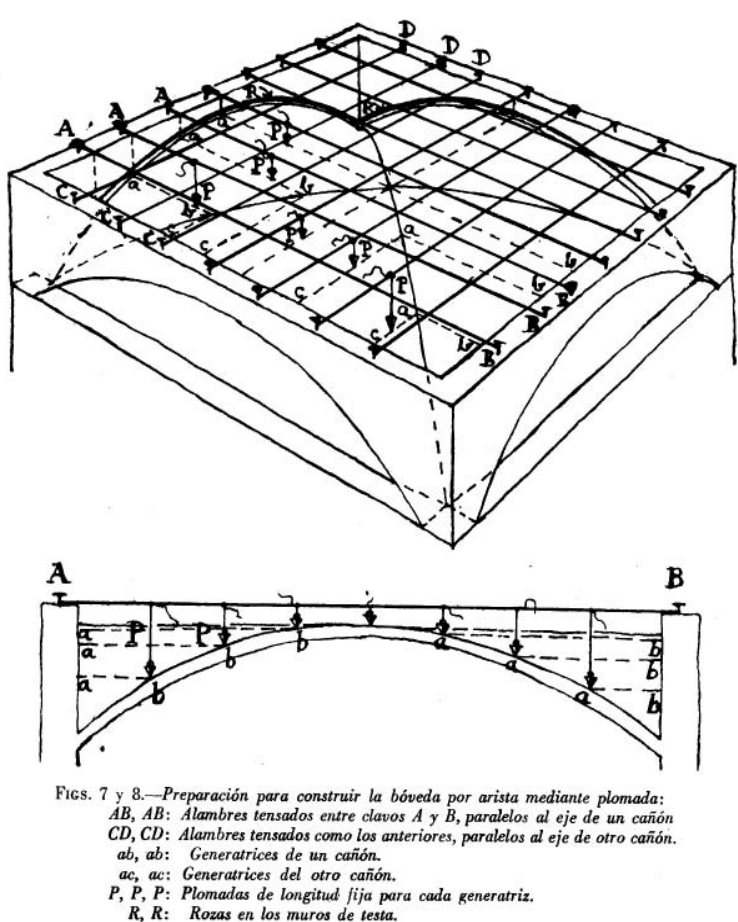

Figs. 7 y 8
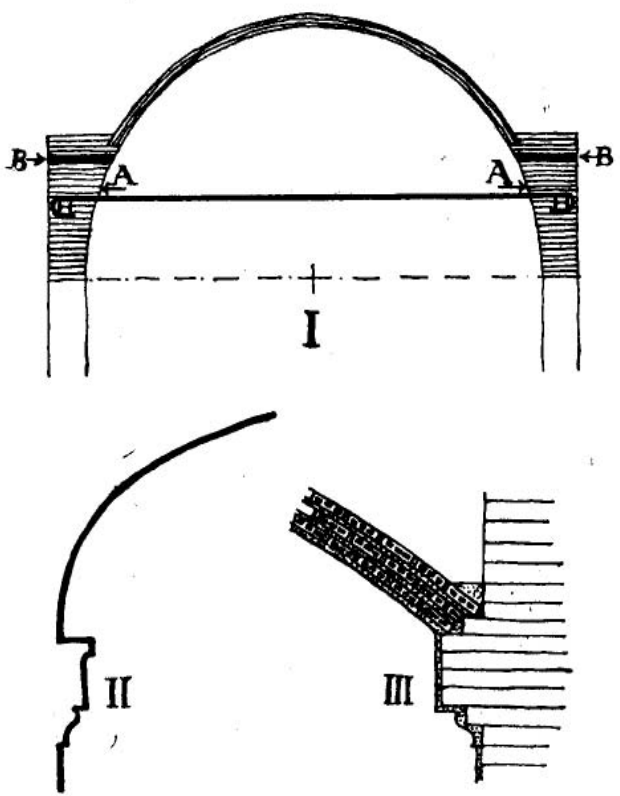

Fig. 16.-I: Bóveda de medio cañón, con arran. que en voladizo.

A: Hiladas de ladrillo macizo en voladizo. $B$ : Piezas metálicas intercaladas entre las hiladas.

II: Arranque de una bóveda maciza antigua, en que la imposta suele servir para apoyo de la cimbra.

III: Arranque de una bóveda de rasilla so. bre hiladas en voladizo para no debilitar el muro de los pisos superiores.
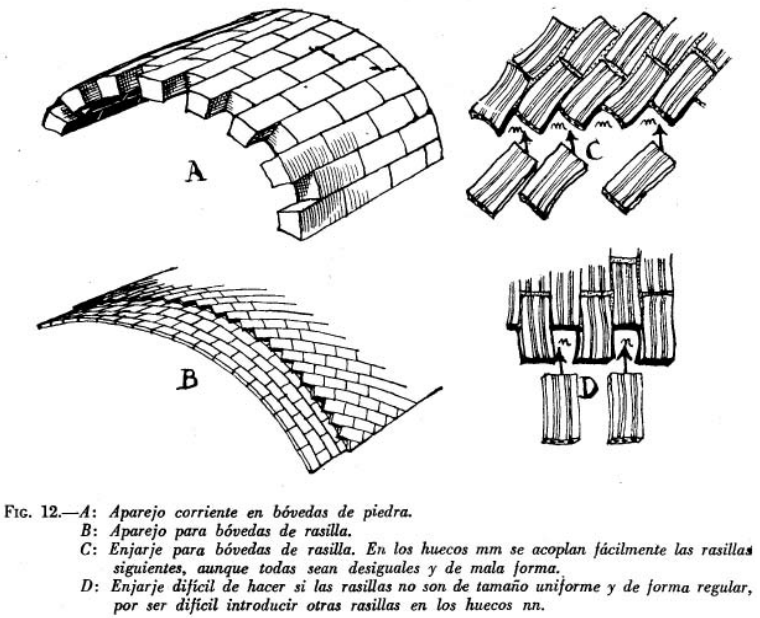

Fig. 12

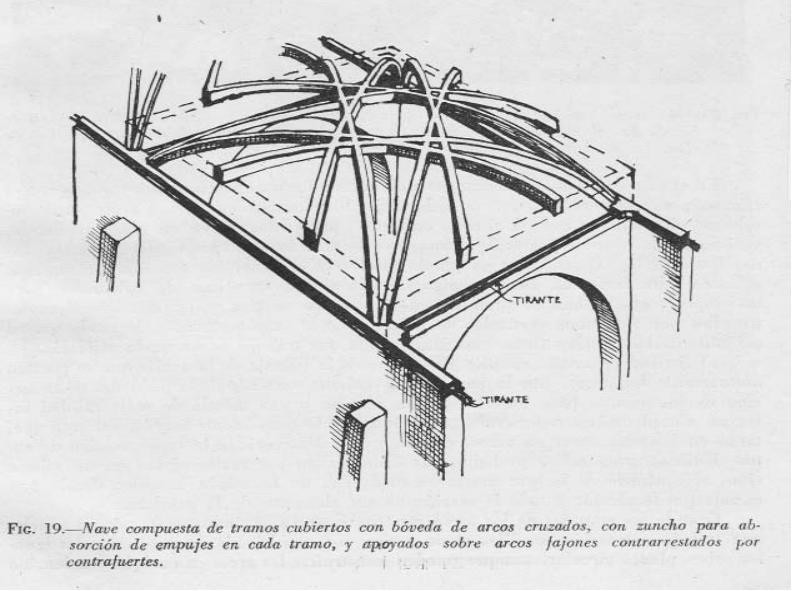

Fig. 19

- Comportamiento de las bóvedas ante diversos movimientos

Luis Moya era perfectamente consciente de los movimientos de las cubiertas abovedadas por efectos térmicos. Sus valores pasan a ser importantes a partir de los $15 \mathrm{~m}$ de luz en la climatología madrileña. Para solucionar este problema, nos expone un detalle de un apoyo de giro y deslizamiento por él diseñado (Fig. 45).

Asientos, vibraciones, dilataciones.

\section{- Trasdosados}

Dada la delgadez de las bóvedas tabicadas, puede peligrar su estabilidad si los esfuerzos a compresión se separan de la directriz curva de la misma, a menos que la bóveda se refuerce con costillas de trasdós, las cuales pueden quedar, la mayoría de las veces, embebidas en el grueso de la 


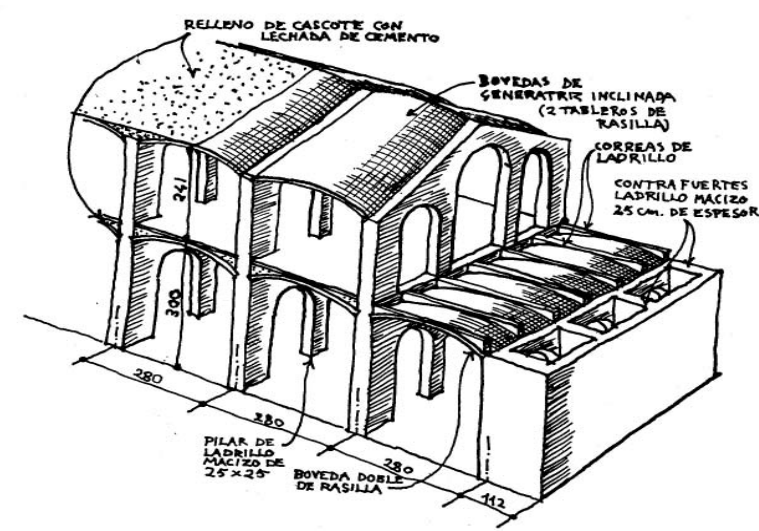

Fic. 25. - Grupo de casas compuestas de dos plantas de bóvedas, contrarrestadas entre si las de cada
planta, y con contrafuertes en los extremo del grupo. Barrio de Usera (Madrid).

Fig. 25

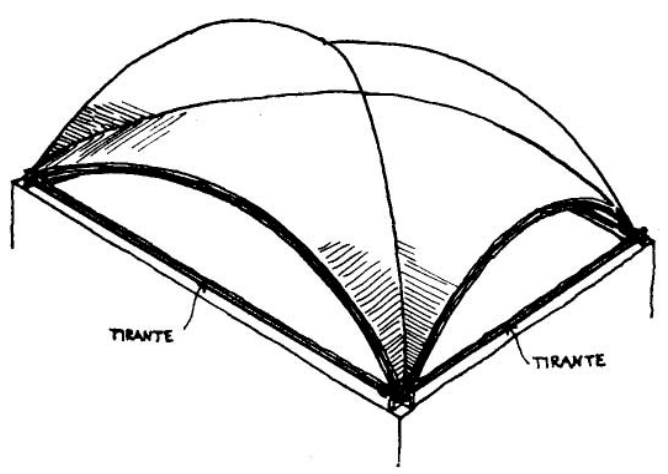

Fig. 36.-Bóveda por arista sobre muros de fábrica con tirantes de hierro redondo ocultos en aquéllos.

Fig. 36

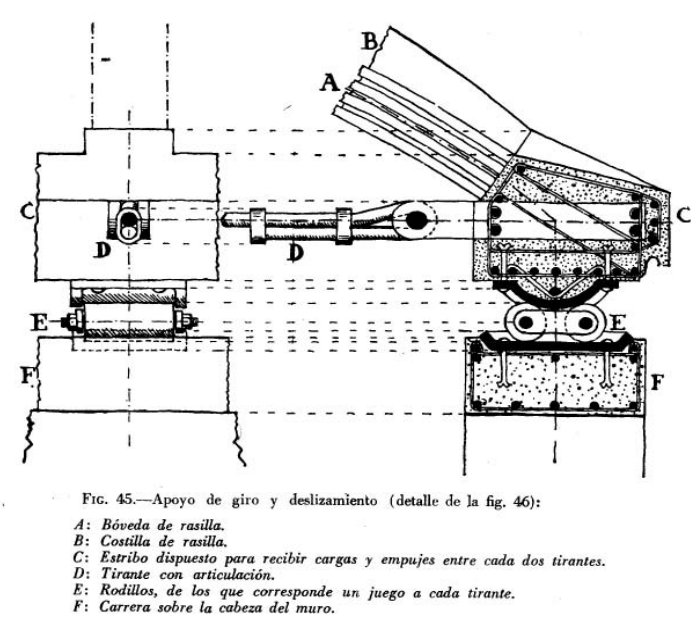

Fig. 45

pendiente de forjado, lo que Luis Moya aplicaba con habilidad aprovechando los huecos entre las costillas, debidamente impermeabilizados, para ajardinamiento de cubiertas planas (Fig.47), lo que expone en los apartados de "bóvedas de cubierta" y "bóvedas para pisos".
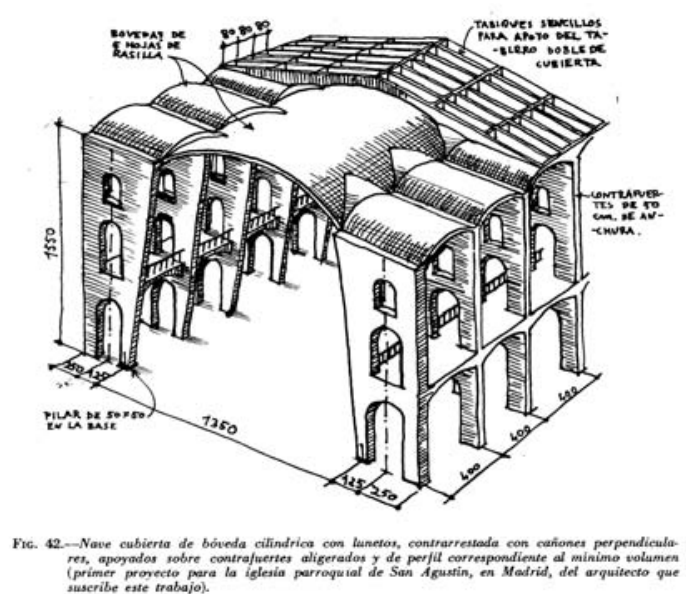

Fig. 42

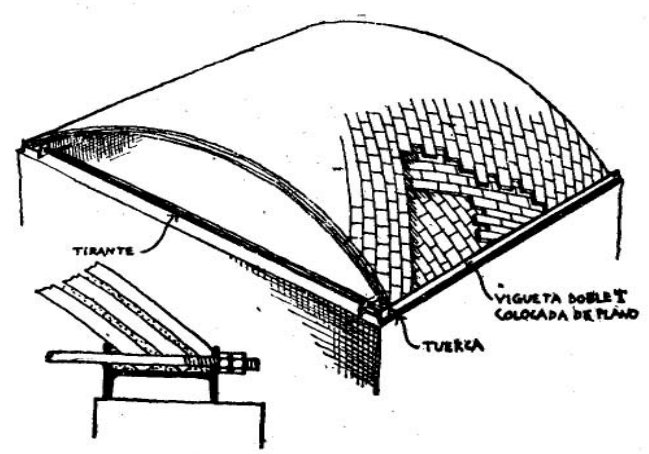

Fic. 37.-Bóveda cilindrica sobre cuadrado o rectángulo, con estribo en los muros laterales y tirante oculto en los muros de testa. El estribo trabaja ca mo una viga apoyada con carga uniforme, que es el empuje de la bóveda.

Fig. 37

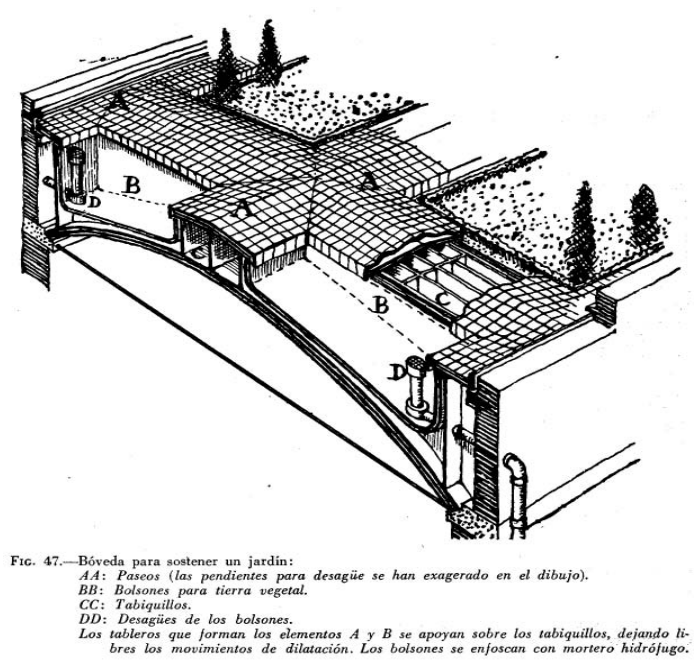

Fig. 47

- Trabajos en el extranjero

El autor del libro, muestra algunos ejemplos del extranjero, destacando a los Guastavino de Estados Unidos, con sus obras del Anfiteatro de la Universidad de Virginia, el Horticultural Hall de Boston, el Hospital de San Lucas de 


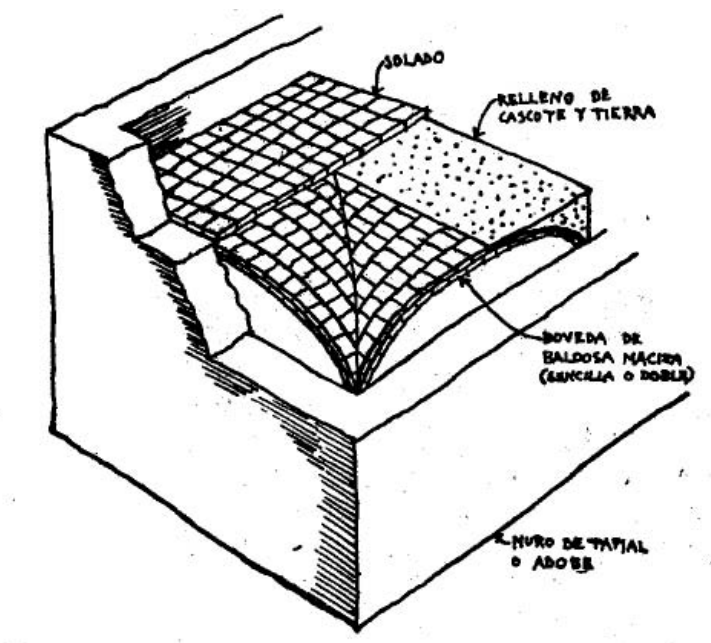

Fig. 69.- Sistema tradicional en Extremadura: $L a$ bóveda se forma de una o dos hojas de baldosa maciza delgada puesta de plano y cogida con yeso. Los muros son de tapial o adobe, $y$ el relleno de cascote y tierra. Se emplea la cal para consolidar el tapial y el relleno.

Fig. 69

Nueva York, las Cuadras de Castlegould en Long Island y la Cúpula del Banco de Montreal de Canadá.

\section{- Bóvedas antiguas}

Respecto de las bóvedas antiguas, se detiene en el análisis de los edificios de la Basílica de Constantino en Roma, Santa Sofía en Constantinopla y la Catedral de Gerona, así como en el Sistema tradicional en Extremadura (Fig.69).
- Datos sobre obras realizadas

Agrupaciones de bóvedas; grupo de casas en el barrio de Usera (Madrid); Iglesia Parroquial de Manzanares (Ciudad Real); Hospital de la Mutual del Clero e Iglesia de San Pedro (Madrid); Museo de América; Iglesia Parroquial de San Agustín.

\section{- Otros edificios posteriores}

Colegio de Nuestra Señora del Pilar; Iglesia de Torrelavega (Santander). Centro Parroquial en Carabanchel; Nuestra Señora de la Araucana en Puerto Rico (Madrid); Universidad Laborar de Gijón; Universidad Laboral de Zamora; Vivienda en Pedro Valdivia; Colegio Mayor Chaminade (Madrid).

\section{BIBLIOGRAFÍA}

- Arte de albañilería o instrucciones para los jóvenes que se dediquen a él. Villanueva, J. de. Madrid, 1827 (edición facsimil por Ediciones Velázquez, Madrid 1977).

- Observaciones sobre la práctica del arte de edificar. Fornés y Gurrea, M. Imprenta de D. Mariano de Cabrerizo. Valencia, 1857 (reeditado por Ediciones Poniente, Madrid 1982).

- Bóvedas Tabicadas. Moya Blanco, L. Ministerio de la Gobernación. Dirección General de Arquitectura. Madrid, 1947.

- Tabicados Huecos. Bergós Massó, J. Ed. Colegio Oficial de Arquitectos de Cataluña y Baleares, 1967.

- Francisco Cabrero, Arquitecto. Xarait Ediciones. Madrid, 1979.

- Curso sobre bóvedas Introducción a las técnicas de ejecución y restauración. Carmona Barrero, J. D. Edita Consultores de Arquitectura y Rehabilitación con la colaboración de la Escuela Politécnica de Cáceres de la Universidad de Extremadura. Almendralejo, 1999. 